\title{
Time-space Visualization of Automobile and Airplane Travel Time from Chicago to Various Destination Cities
}

Stella W. Todd Metropolitan State University of Denver, toddst@msudenver.edu* , Alex Yetsko Metropolitan State University of Denver, ayetsko@blm.gov, Claire Hay University of Colorado, Denver, claire.hay@ucdenver.edu

\footnotetext{
* Corresponding author
}

\begin{abstract}
Travel distances are often thought of in terms of time. When asked about how far away a destination is, we answer that it is " $x$ " minutes or hours away. This study explores a novel approach to visualize travel time from a selected, specific city to various destination cities in the Conterminous United States using two modes of transportation: automobile and airplane. Each destination city was selected randomly from cities that met a set of criteria including: city population categories, state representation, and overall geographic distribution. For the automobile and airplane time-space visualization Google Maps were consulted to get driving time and flying time estimates to 107 destination cities. For the airplane travel additional time was added to estimate driving to a local airport and parking. A straight-line timevector trajectory between the example start city, Chicago, and the destination cities provided a consistent line to alter the relative locations of destination cities relative to buffer distances representing time. Timealtered city locations were used as control points to align an existing map of the United States. The automobile results show pronounced geographic dilation as well as contraction along many coastal states. Airplane travel severely contracted geographic extent of the U.S. as flying time is much faster and far less variable than automobile time. These visualizations provided a different cartographic product that emphasizes the time and space perception changes in time and space associated with movement via different modes of travel.
\end{abstract}

Keywords: time visualization, time-space, time cartogram, relative time, perception, travel time

\section{Introduction}

The concepts of location, direction, distance and time are interrelated within a geographic, spatial perception of time and space. We know this through experience as we navigate from one place to another. Time and space have different frames of reference and different units of measurement. For the normal, human observer the relationship between time and space varies with speed and direction of the observer relative to the objects speed and direction. Time perception and space perception are intertwined within change perception. Visualization, i.e., the creation of maps of human perception of the time-space relationship in which we exist in our everyday lives helps us to better contextualize our personal travel experience.

\subsection{Time and Space Perception and Cognition}

The earliest pre-historic maps were figurative drawings that represented locality (Wolodtschenko and Forner, 2007). Mental constructions of spatial experience are still important in cartography. Mental maps record our spatial memories that vary with our physical limitations, preferences, knowledge, as well as "compendiums of all things worth knowing" (Gould and White, 1986; Edlund, 2018). The perception of change depends on movement that can be described through speed, direction, and varied locations. Space and time are 
unified through change perception (Khurana and Nijha, 2010).

Spatial cognition of our environment involves mental maps of space that are well known to human experience. The psychological and physiological mechanisms of this construction are beginning to be understood. Using a regular grid pattern of brain cell firing rats create a grid view of space, through sensory cues that are subsequently modified through experience (Carpenter et al., 2015). A system of brain cells have been discovered in rats that fire along a continuous grid pattern that responds to both direction and speed of movement. Independent spatial grid patterns become merged when unconnected experiences become connected (Carpenter, et al., 2015). Similar grid cells patterns have been identified for primates, with a triangular network of firing locations that respond to eye movement across space (Killian et al., 2012). Discretized (raster data models) representations are more than just abstract models of space but are actually similar to mammalian mental maps.

Advancements in navigation and geodesy changed our spatial reference to an earth-centered one first described by Ptolemy (Berggren and Jones, 2000). Technological advances continue to make locating positions on earth more precise and more accurate. Map projections display these positions on an XY coordinate plane that maintains accuracy in one or more properties that include shape, size, distance, and direction. While real-world $2 \mathrm{D}$ and $3 \mathrm{D}$ spatial coordinate systems dominate cartography a recent emphasis on time-space interrelationships as a desirable attribute in a map is understandable given our fast-paced ever-changing societies and landscapes.

\subsection{Time and Space Visualization}

Time and space are intertwined. Time is thought to be a single dimension by most physicists. Time and space can be visualized jointly in 2 to 4 dimensions. Time coordinates are incorporated in time geography depictions of life path experiences where space is assigned to the $X$ axis and time to the $Y$ axis (Hagerstrand, 1970). While this method depicts time and distance, it generalizes direction to either away from or toward a home base starting location. This method depicts movement over time but not exact change of position over time. Time is incorporated as a $3 \mathrm{rd}$ dimension relative to a $2 \mathrm{D}$ landscape or as the fourth dimension in conjunction with a 3D landscape.

Geographic information systems are capable of various models of temporal and spatial patterns such as moving objects, moving patterns of clusters and trajectories, semantic enrichment of timeoriented data, time database attributes, time labels, timestamps, baselines, image series, spacetime cubes, real-time rendering of dynamic scenes (Zhong et. al, 2012).

Cartographic products depicting the dynamics of a time dependent variable typically start with spatial displays and then incorporate time as an element using a variety of methods. For example time may be displayed as a $Z$ coordinate, time sequence of spatial coordinates, or $\mathrm{XYZ}$ time vector lines (Brownrigg, 2005; Slocum et. al., 2009). Raster models can represent the $Z$ dimension as a surface volume. Time can also be displayed as an attribute data value associated with a specific point, line, or area such different emergency response times for a transportation feature (ESRI, 2007). Sequences of images or feature positions may be viewable as animations, small multiples of time, or change maps (Tufte, 1990).

Historically, travel trajectories have connected space and time. A gravity model representing population distribution and growth implements Tobler's first law of geography Miller, 2004). Geometric networks or network datasets may contain time variables such as impedance or speed, and speed limits which helps to predict time to traverse segments of the network (Price, 2009).

Cartograms provide a unique approach to visualizing variables in a spatial context. $A$ cartogram is a map in which actual spatial relationships, e.g., area or distance, are distorted based upon a statistical variable that is used to transform the spatial $X Y$ axes based upon the value of that statistical variable. The advantage of cartograms is that they emphasize data values and deemphasize spatial position. They warp spatial 
coordinates of lines or areas based on thematic variables.

Examples of distance (or linear) cartograms involve warping user-centric linear travel network space relative to time values (Kaiser et al., 2010) or maintaining fixed vertex positions while distorting network lengths (Buchin, 2014). A Linear cartogram visually represents the distance between two locations based upon travel time between the locations. The underlying base map is deformed in such a way that the travel-time vector is substituted for the Euclidean distance vector between the two locations. Value-by-area cartograms scale the map feature areas based on values of a chosen attribute (Dent, 1975). For continuous area cartograms, rubber-sheeting algorithms apply to each polygon from a centroid point (Douginik et al, 1985). For example, area cartograms may show duration or time requirements such as average lifespan or years of education. Table cartograms start with equalsized rectangles (Evans et al., 2018). Cartograms with mathematical morphology can preserve the global shape while varying the local areas and shapes (Sagar, 2014).

\section{Main body}

Often travel distances are thought of in terms of time. When asked how far away a destination is, we answer that it is " $X$ " minutes or hours away. This study explored ways to visualize travel time from the perspective of a single starting city to various destination cities in the Conterminous United States. The overall objective of this project was to visualize the user-centric perceptual experience of automobile travel relative to airplane travel based on travel time by using a combination of linear cartograms and areal transformations.

Superimposing time coordinates on top of distance coordinates helped us view changes in distance relative to time differences between automobile and airplane travel. The approach allowed to warp space to coincide with time-based coordinates in order to visualize perceived temporal differences between travel by automobile and airplane. A linear cartogram of time vectors provided control point locations for time-based spatial rectification and resampling process for the global shape of the conterminous United States. This type of transformation process (called alignment, registration, georeferencing, or rectification) is typically available within geographic information system or image processing software packages. The resulting maps emphasized possible differences in space perception of travel between the automobile and airplane.

\subsection{City Selection Process}

The primary cities and roads were from an ESRI, Inc. 2010 dataset. The spatial context for this project was the conterminous United States and the modes of transportation the automobile and commercial jet aircraft. Chicago was chosen as the starting city and each possible destination city as separate events (Fig. 1).

Sample destination cities were selected from the 35,432 cities dataset based on the following location and demographic criteria: 1) proportion of cities selected from the eastern and western regions of the Conterminous US was approximately the same, 2 ) only cities with a reported population based on the 2000 census were selected, 3 ) an additional eight large cities ( $>500,000$ population in 2000) were randomly selected as destination cities, 4) cities less than 80 kilometres from another city were not selected (manual process), 5) additional cities close to the coastline were included in order to generate contour intervals for the entire US and to produce a map with recognizable boundary features, 6 ) additional cities were added to have representative cities in most states (48 of 49). These cities had a population over 30,000, and 7) when the route between Chicago and more than one destination city included passage through an intermediate city that city was frequently added as a destination as well. Although the road network was not visually modelled in this study it was used to estimate time and distances for automobile travel. The city selection process resulted in 107 destination cities being selected.

An azimuthal projection was chosen that is equidistant with the Central Meridian being close to the geographic center of the Conterminous United States and selected cities were merged into 


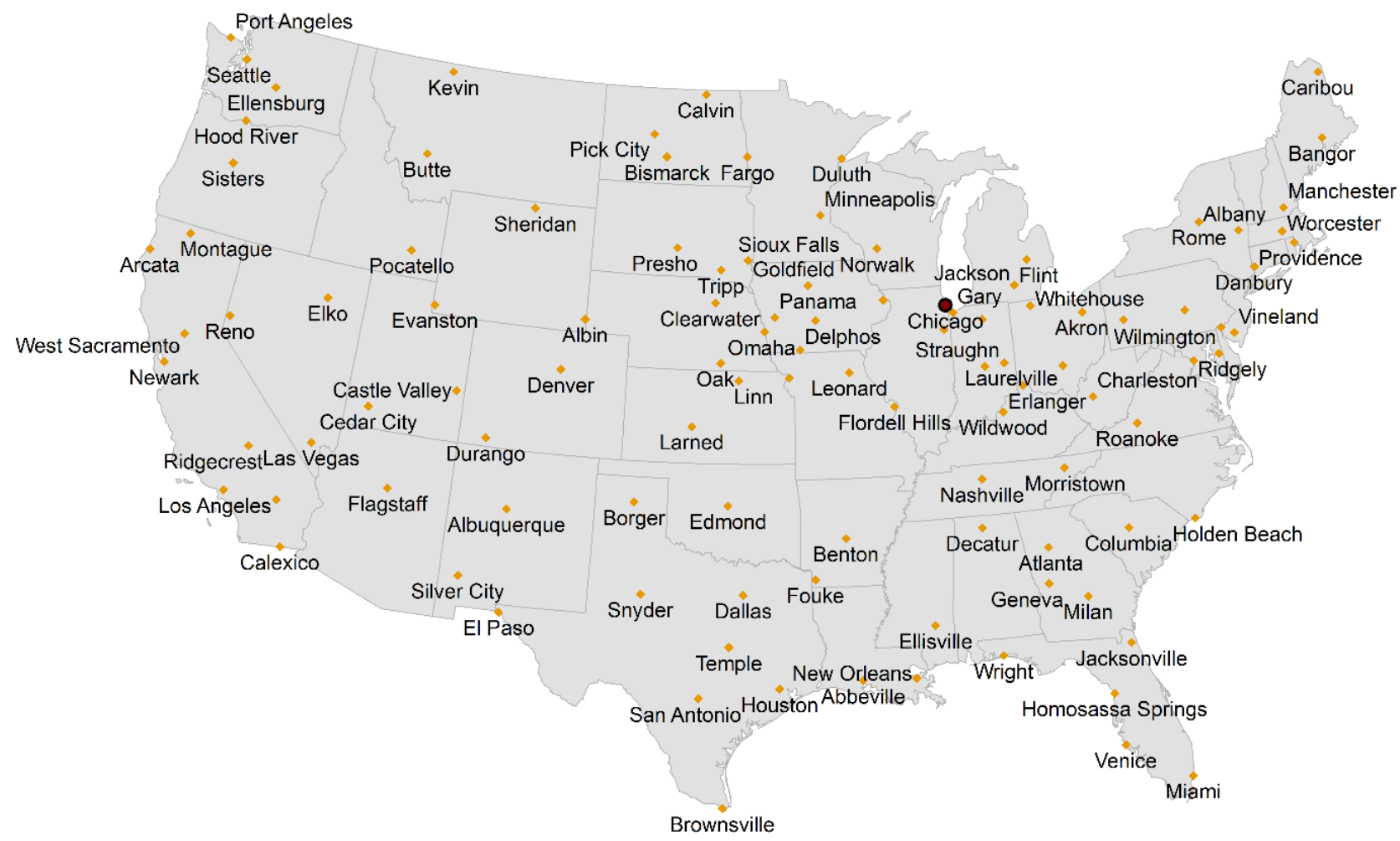

Figure 1: Chicago and 107 destination cities of various population sizes

a single dataset. All maps were produced at a 1:30,000,000 scale.

\subsection{Visualization Methods:}

Two visualization methods were compared: 1 ) visualization of time-relative distance using actual driving time and 2) visualization of time-relative distance using actual airplane and transfer time. Both of the time-based spatial adjustment methods used a straight-line time-vector representation between the start city and the destination cities. The direction-vector provided the orientation relative to Chicago from which to adjust distances of destination cities. A time-based coordinate system was created from Chicago by creating equally spaced time buffers to a half-hour precision. The selected destination cities were assigned time-based coordinates along the orientation vector direction between them and Chicago (Fig. 2).
2.3 Visualization of Time-relative Distance Using Actual Driving Time

Time (minutes) and distance (meters) to destination cities were determined using Google Map data of travel time with Chicago as the origination city. The fastest route was chosen if alternative routes were indicated. Using the selected cities a polygon feature class of buffer distances at one-half hour increments was created. Cities were manually relocated along the vector line to intersect the buffer line most similar to the Google Time for that destination city. A conterminous states .tiff file was transformed to correspond with the temporary relocated city coordinates using an alignment (register raster) function that is typically used for georeferencing raster layers with unknown or incorrect coordinate locations using ground control points from known correct locations. The relocated city coordinates provided the ground control locations for a link file of from and to $X Y$ coordinates used in a spline interpolation of the conterminous states file. 


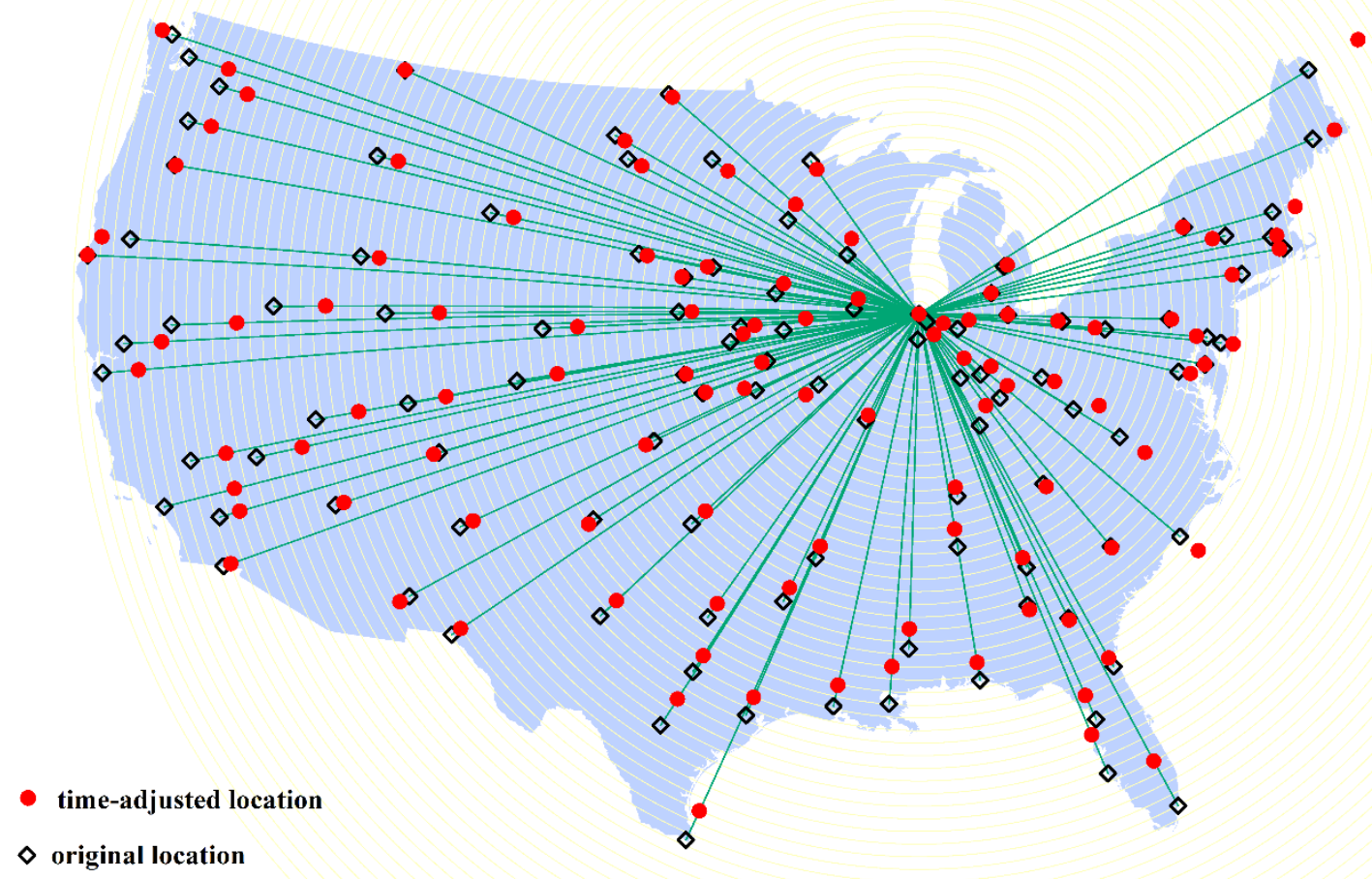

Figure 2. Destination cities from Chicago, their vector directions, half-hour time buffers, and automobile time-adjusted locations

\subsection{Visualization of Time-relative Distance Using Actual Airplane and Transfer Time}

The flight-time data to each city was gathered from Google Maps with Chicago as the starting point and each selected city as the destination. If the cities had nonstop or connecting flights from Chicago those flight times where entered in to the table. If, however, there is no airport within the city the flight time to the closest airport was entered and then the drive time from that airport to the destination city was added to the flight time. The drive time was used for any city where drive time was faster than flight time. Once the data table was completed two hours was added to each city that had flight data to account for ground-based transfer time such as boarding and disembarking from the aircraft as well as any other ground-based activities, such as renting a car or parking. Within an ArcGIS editing session, each city was manually relocated along its orientation vector to the appropriate half-hour buffer isoline.
Once the linear cartogram of travel time was produced it was possible to transform the global area of the conterminous United States. After each destination city was moved to their appropriate isoline a .TIFF image of the cities in the Conterminous United States was created. Next a continuous surface was interpolated using the same alignment methods as were used for the automobile data using the new temporary city locations as $\mathrm{XY}$ control points linked to the original city coordinates with a spline transformation.

\subsection{Results}

No independent time scale exists away from a specific reference location thus all city relocations are relative to that specific reference point. When comparing absolute driving times the city of Arcata had the longest travel time from Chicago. But, it was also far away. Here the moderate time adjustment away from Chicago was partly due to the expanse of the Sierra Nevada mountain range in California. Because time-buffers were concentric around Chicago different cities (some eastern and 
southern cities) were relocated off-shore even though they had shorter times to Chicago than Arcata, CA. Examples such as Brownsville, TX Holden Beach, NC and Caribou, ME were associated with smaller highway networks typical of regions with smaller populations. Some Cities along the west coast of the US such as Los Angeles, CA and Seattle, WA were notably relocated closer to Chicago using absolute time (Fig. 3a). These cities are located along interstate highways.

The extensive development of interstate highways within the United States makes average automobile velocity fairly consistent between large cities. Smaller highways and minor roads have more variable speed limits. Therefore cities that connect entirely by interstate highway networks are temporally closer together relative to those lacking complete interstate access. Mountainous regions are slower than flatlands, even with interstate highway access. The combination of physiographic features and road development type largely determines relative travel time.

The difference between driving and flying time representations is dramatic (Fig. 3b). Although the splined surface was using both the driving and flying representations the cartogram display of states is much more distorted in the flight-time instance. This is due to a few cities with very long travel times due to the lack of proximity to an airport. For example the mean travel time was 5.6 hours with a standard deviation of 2.2 hours. Calexico, CA and Worcester, MA were difficult to get to from Chicago with flying and transfer times of 13.8 and 14.4 hours respectively. Less evident from the spline surface are a few cities that were relocated farther inward toward Chicago than other cities because of very fast travel speeds. For example, the mean flying travel speed was 295.7 $\mathrm{km} / \mathrm{hr}$ with a standard deviation of 131.1.

Southwestern cities such as Las Vegas, NV, West Sacramento, CA, Reno, NV, Twentynine Palms, CA, and Seattle, WA had travel speeds in excess of 535 $\mathrm{km} / \mathrm{hr}$. These cities cause the cartogram display to fold inward towards Chicago.

\section{Conclusion}

Explicit displays of time were demonstrated in this study that intertwined the spatial and temporal dimensions in a unique way to emphasize the impact of time on spatial perception. For people living in Chicago it is simply easier to travel long distances by automobile to the southwest and south rather than the northeast or northwest. The areal differences between airplane and drive times dramatically illustrate that space shrinks when travel time is significantly shortened. The perception of change in location from an airplane depends in part on whether or not the passenger observes changes in the landscape changes while looking out the window. Otherwise the transition from one location to another may seem like sitting in a room that tilts and sways.

Mapping time spatially may be helpful in understanding travel perceptions and visitation patterns particularly for discretionary travel. Mapping travel time as a planar grid surface is difficult because the origin needs to be flexible relative to a start time. While the US visualizations of time in this study show some notable regional patterns relative to Chicago the well-developed highway system modulates the time variations somewhat. Time variation might be a much larger factor in less-developed countries where road construction is more variable. Tourist perception of time/space relationships will strongly limit or encourage the visitation of specific landmarks.

Travel driving speeds vary as a consequence of numerous factors such as: regional differences in posted road speeds, road construction and maintenance, landforms, travel habits and weather conditions across the United States. No statistical relationship between driving time and population or road network density was apparent through simple graphing procedures. Flight times also vary as some regions have well-established airport systems and others have less access to flights and flight connections.

Calculations of average travel time from a city to other locations can only be done specific to a single starting location. A more automated process where such analyses could be carried out for 

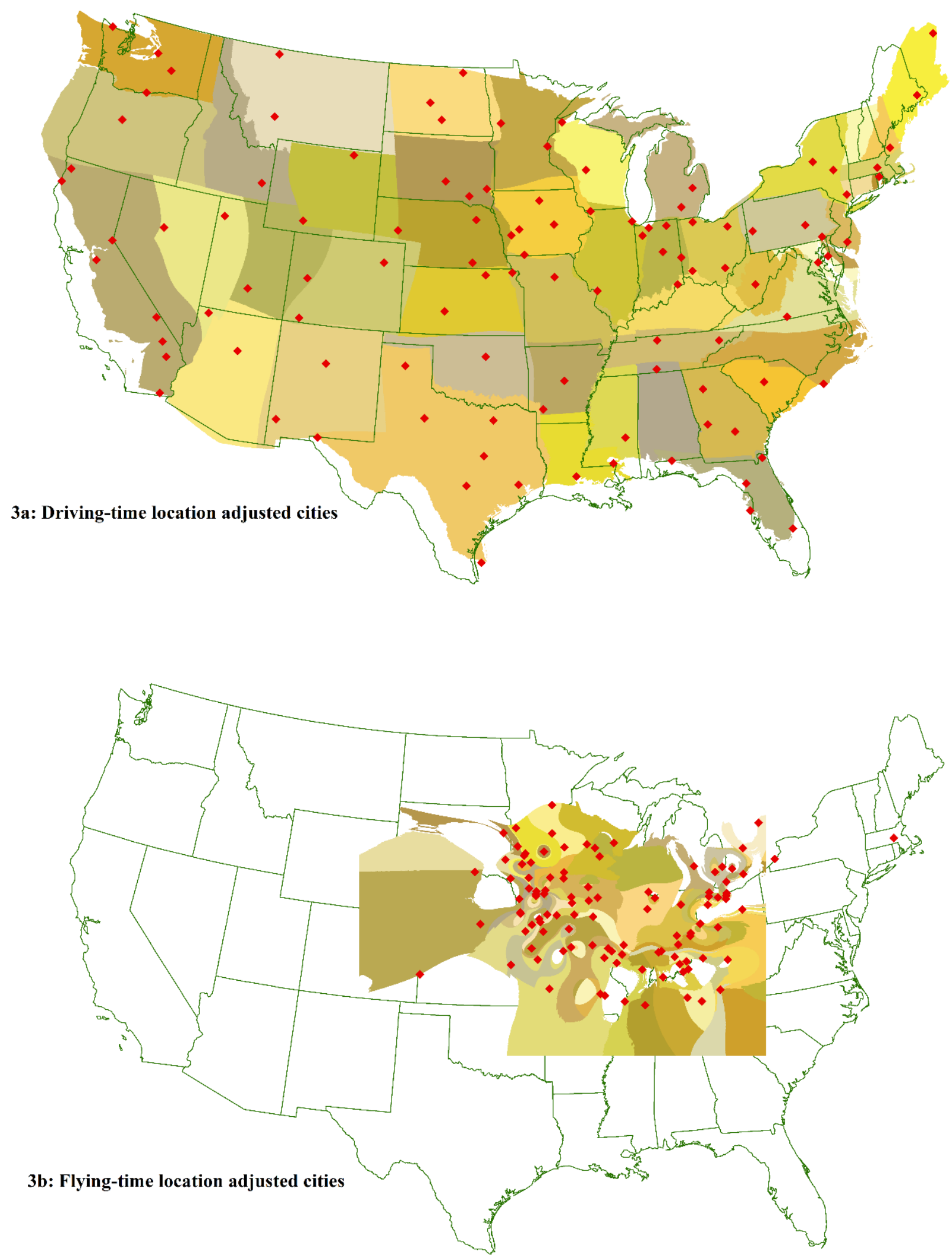

Figure 3: Georeferenced spline interpolation cartograms

Proceedings of the International Cartographic Association, 2, 2019.

29th International Cartographic Conference (ICC 2019), 15-20 July 2019, Tokyo, Japan. This contribution underwent

single-blind peer review based on submitted abstracts. https://doi.org/10.5194/ica-proc-2-131-2019 | ๔ Authors 2019. CC BY 4.0 License. 
multiple starting cities to multiple destinations cities could be useful to develop a connectedness measure for world cities.

\section{Acknowledgements}

Thanks to Benjamin Dixon for his contributions to airplane time travel calculations. This paper was supported by Metropolitan State University of Denver sabbatical funds.

\section{References}

Berggren, J. L. and Jones, A. (2000). Ptolemy's Geography: An Annotated Translation of the Theoretical Chapters. Princeton University Press, Princeton.

Buchin K., van Goethem A., Hoffmann M., van Kreveld M., Speckmann B. (2014) Travel-Time Maps: Linear Cartograms with Fixed Vertex Locations. In: Duckham M., Pebesma E., Stewart K., Frank A.U. (eds) Geographic Information Science. GIScience 2014. Lecture Notes in Computer Science, vol 8728. Springer, Cham

Carpenter, F., Manson, D., Burgess, N., and Caswell, B. (2015). Grid Cells Form a Global Representation of Connected Environments. Current Biology, 25:1176-1182

Daya Sagar, B.S. (2014). Cartograms via mathematical morphology. Information Visualization 2014, Vol 13(1) 42-58

Dent, B. D. (1975). Communicative aspects of valueby-area cartograms. American Cartographer, 2, 15-68

Dougenik, J. A., N. R. Chrisman, and D. R.

Niemeyer (1985). An algorithm to construct continuousarea cartograms. The Professional Geographer 37 (1): 75-81.

ESRI (2007). GIS for Fire Station Locations and Response Protocol: An ESRI White Paper. ESRI, Inc, Redlands, 28 pgs.

Edlund, L (2018) Some reflections on mental maps, Journal of Cultural Geography, 35:2, 274-285

Evans, W., Felsner, S., Kaufmann, M, Kobourov, S.G., Mondal, D., Nishat, R. I. and Verbeek, K. (2018). Table Cartogram. Computational Geometry 68 (2018): 174-185
Gould, P., and White, R. (1986). Mental Maps 2nd Ed., Penguin Books

Hagerstrand, T. (1970). What about People in Regional Science? 9th European Congress of the Regional Science Association, pp 7-21.

Kaiser C., Walsh, F., Farmer C. J. Q., Pozdnoukhov, A. (2010) User-Centric Time-Distance Representation of Road Networks. In: Fabrikant S.I., Reichenbacher T., van Kreveld M., Schlieder C. (eds) Geographic Information Science. GIScience 2010. Lecture Notes in Computer Science, vol 6292. Springer, Berlin, Heidelberg

Killian,N.J., Jutras, M.J. and Buffalo, E.A. (2012). A map of visual space in the primate entorhinal cortex. Nature advance online publication

Miller, Harvey J., (2004) Forum: Tobler's First Law and Spatial Analysis. In Annals of the Association of American Geographers, 94(2), 2004, pp. 284289

Price, M. (2009). Do it Yourself!: Building a network dataset from local agency data. ArcUser, Summer, 2009, ESRI, Inc.

Slocum, T.A., McMaster, R.B., Kessler, F. C., and Howard, H.H. (2009). Thematic Cartography and Geovisualization, 3rd Ed, K.C. Clarke, editor

Tobler W., (1970) A computer movie simulating urban growth in the Detroit region. Economic Geography, 46(Supplement): 234-240

Tufte, E. (1990). Envisioning Information. Graphics Press, Cheshire, CT

Wolodtschenko, A., and Forner, T. (2007).

Prehistoric and Early Historic Maps in Europe: Conception of Cd-Atlas. e-Perimetron, Vol. 2, No. 2, Spring 2007, 114-116

Zhong, C., Wang, T., Zeng, W., and Arisona, S.M. (2012). Digital Urban Modeling and Simulation: Communication in Computer and Information Science, 242: 299-317 